\title{
Improved Greedy Algorithm Based Matrix Satellite Cable Connection Optimal Design
}

\author{
Yiyi Wang ${ }^{1}$, Zhifu Wang ${ }^{1}$, Ke Zong ${ }^{1}$, Mian Ye ${ }^{1}$, Xi Zhou ${ }^{1}$ and Zhijie Wang ${ }^{1}$ \\ ${ }^{1}$ Institute of Telecommunication Satellite, China Academy of Space Technology, Beijing 10094,China
}

\begin{abstract}
An improved greedy algorithm based matrix satellite cable optimal design method is presented in this paper aim to overcome the difficulties including designing complexity, designing cycle and iterative designing process. An optimal algorithm model is built to help designer not only to accomplish design correctly and efficiently, but also to obtain better designing outcomes. Firstly, matrix cable designing model is pre-translated by turning electrical interface to equivalent digital model. Then the digital model is turned into computer language. At last, optimal design rules and method are extracted according to improved greedy algorithm. Real project data are used to prove the feasibility and validity of the proposed method.
\end{abstract}

\section{Introduction}

Matrix circuit including matrix telemetry and command is a core technology of on-board integrated electronic technology [1] to reduce the amount of electronic components and cables. This technology is widely used in all kinds of satellites to observably lose the weight of satellite platform. The devices with matrix telemetry and command can be connected with integrated electronic subsystem by matrix cable to send commands and receive telemetries. Matrix cable design has great possibility to be improved by reducing the usage of cables because matrix circuit usually has a large number of brunches which may cause the redundancy of design.

Consequently, an improved greedy algorithm based matrix satellite cable optimal design method in presented in this paper aim to overcome the difficulties listed above. Computer based design can acquire correct and preferred outcome, meanwhile, improve the work efficiency.

\section{Matrix cable contact design principle}

Matrix command contact circuit comprises command drive device and command load device. Command drive device can control $\mathrm{m} \times \mathrm{n}$ pieces of load device by control $\mathrm{m}$ row cables and $\mathrm{n}$ column cables ${ }^{[2]}$. It takes $2(\mathrm{~m}+\mathrm{n})$ cables to control $m \times n$ pieces of load device if backup is included. Matrix telemetry contact circuit is using the same principle. Matrix cable contact design principle is to build a point-to-point mapping relation between drive devices and load devices. By building a physical cable connection, drive devices can control load devices to executive commands and receive telemetries.

\section{Improved greedy algorithm based satellite matrix cable connection optimal design}

\subsection{Design model pre-processing}

Circuit model is tuned into structural digital model to achieve optimal design. Assuming that a command load device has 9 matrix commands controlled by 3 row cables and 3 column cables, it can be shown as $\mathrm{M}_{\mathrm{RC}}=\left[\begin{array}{lll}M_{11} & M_{12} & M_{13} \\ M_{21} & M_{22} & M_{23} \\ M_{31} & M_{32} & M_{33}\end{array}\right]$. be shown as $\mathrm{N}_{\mathrm{RC}}=\left[\begin{array}{ccc}N & \cdots & N \\ \vdots & \ddots & \vdots \\ N & \cdots & N\end{array}\right]_{R \times C}$.

From the knowledge mentioned above, row 1 and column 1 take control of M11. According to the principle, circuit connection can be translate into mapping relation between drive device model and load device model. If $\mathrm{NRC}=\left[\begin{array}{ccc}N & \cdots & N \\ \vdots & \ddots & \vdots \\ N & \cdots & N\end{array}\right]_{5 \times 5}$, the relation can be shown as $\left[\begin{array}{lllll}N & M & M & M & N \\ N & M & M & M & N \\ N & M & M & M & N \\ N & N & N & N & N \\ N & N & N & N & N\end{array}\right]$.

\subsection{Formula expression of design model}

\footnotetext{
* Corresponding author: wyywangyiyi@126.com
} 
Formula expression of design model is $\{\mathrm{Rx}, \mathrm{Cy}, \mathrm{k}\}$.

$\mathrm{Rx}$ represents for row cable, Cy represents for column cable, $\mathrm{k}$ represents for the number of matrix model on one device.

\subsection{Improved greedy algorithm based matrix cable connection optimal design method}

Optimal design is achieved by algorithm based programming on the basis of digital model and formula expression input.

A greedy algorithm does not consider the overall result, but aim to find a partial optimum. In most cases, a greedy strategy follows an optimal principle and takes partial optimum step by step. The greedy algorithm structure is as follows.

Beginning with an original solution for a problem,

While ( making one step forward to final goal)

\{

Use available strategies to find a solution

Get a final solution by combining all the solutions

VLSI routing problem ${ }^{[3]}$ and 2D packing problem ${ }^{[4]}$ chooses different row cable and column cable will get different matrix connections. Travelling salesmen problem ${ }^{[5]}$ has capacity restriction and mutual restriction. Unlike VLSI or TSP problem, this case is a $2 \mathrm{D}$ vehicle routing problem [6] with confliction and capacity restriction. To solve this problem, this article raised an improved greedy algorithm with complexity of $O\left(M^{*} n\right)$. $\mathrm{M}$ represents the maximum of row or column, $\mathrm{N}$ represents the amount of matrix.

\subsubsection{Optimal Design rules summary}

Step 1 List all Matrix signals. $\{\mathrm{T}\}$ is the set of telemetries; $\{\mathrm{C}\}$ is the set of commands. $\left\{\mathrm{T}_{\mathrm{M}}\right\}$ is the set of matrix telemetries; $\left\{\mathrm{C}_{\mathrm{M}}\right\}$ is the set of matrix commands. $T_{M} \in T, C_{M} \in \mathrm{C} .\left\{\mathrm{T}_{\mathrm{M}}\right\}$ and $\left\{\mathrm{C}_{\mathrm{M}}\right\}$ should be managed separately.

Step 2 Separate all devices into different secions. Put devices into correspoding section lists by recognize their coordinates.

$$
T_{M}\left\{\begin{array}{l}
\text { South Part }\left\{\begin{array}{l}
+X \\
-X
\end{array}\right. \\
\text { North Part }\left\{\begin{array}{l}
+X \\
-X
\end{array}\right.
\end{array}\right.
$$$$
C_{M}\left\{\begin{array}{l}
\text { South Part }\left\{\begin{array}{l}
+X \\
-X
\end{array}\right. \\
\text { North Part }\left\{\begin{array}{l}
+X \\
-X
\end{array}\right.
\end{array}\right.
$$

Definition $1 \mathrm{~T}_{\mathrm{Max}}$ is the matrix telemetry with maximum of row or column in $\left\{\mathrm{T}_{\mathrm{M}}\right\}$.

Definition $2 \mathrm{C}_{\mathrm{Max}}$ is the matrix command with maximum of row or column in $\left\{\mathrm{C}_{\mathrm{M}}\right\}$.
Definition $3 \mathrm{~T}_{\text {Sum }}$ is the matrix telemetry with maximum sum of row and column in a specific set of telemetries.

Definition $4 \mathrm{C}_{\text {Sum }}$ is the matrix command with maximum sum of row and column in a specific set of commands.

Step 3 Find TMax and $\mathrm{C}_{\text {Max. }}$. If there are more than one $\mathrm{T}_{\text {Max }}$ or $\mathrm{C}_{\text {Max }}$, we get $\left\{\mathrm{T}_{\operatorname{Max}}\right\}$ or $\left\{\mathrm{C}_{\text {Max }}\right\}$. Then we find $\mathrm{T}_{\text {Sum }}$ or $\mathrm{C}_{\text {Sum. }}$. If there are more than one $\mathrm{T}_{\text {Sum }}$ or $\mathrm{C}_{\text {Sum }}$, the priority is random.

Rule 1 Using row cable is prior to column cable if the total increment and partial increment are fixed.

Definition 5 If one device has been appointed to be $\mathrm{D}_{\text {Aim }}$, algorithm cannot move on to other device until all the matrix signals are allocated.

Definition 6 The set of all matrix telemetries of $\mathrm{D}_{\text {Aim }}$ except $T_{\text {Max }}$ is $T_{\text {Next. }}$ The set of all matrix commands of $\mathrm{D}_{\text {Aim }}$ except $\mathrm{C}_{\text {Max }}$ is $\mathrm{C}_{\text {Next. }}$.

Step 4 When $\mathrm{T}_{\text {Max }}$ or $\mathrm{C}_{\mathrm{Max}}$ is chosen, the device $\mathrm{T}_{\text {Max }}$ or $\mathrm{C}_{\text {Max }}$ becomes $\mathrm{D}_{\text {Aim. }}$. $\mathrm{T}_{\mathrm{Next}}$ of $\mathrm{C}_{\mathrm{Next}}$ must be assembled with $\mathrm{T}_{\mathrm{Max}}$ or $\mathrm{C}_{\mathrm{Max}}$ to get the minimum sum of rows and columns in order to use cables as less as possible.

Definition 7 The set of devices on the same branch of $D_{\text {Aim }}$ except $D_{\text {Aim }}$ is $\left\{D_{\text {Next }}\right\}$.

Definition $8 \mathrm{~T}_{\text {Aim }}$ or $\mathrm{C}_{\text {Aim }}$ is the matrix signal which is going to be allocated then.

Step 5 When finished $D_{\text {Aim, }}$ repeat step 3 and 4 for $\left\{\mathrm{D}_{\mathrm{Next}}\right\}$.

Rule 2 The second junction point is the flag of where a branch starts.

Rule 3 If there are more than one position with minimum cost increment, length increment is the solution to find the best choice.

Step 6 When $\left\{\mathrm{D}_{\mathrm{Next}}\right\}$ is finished, repeat step 1 to 5 for the rest.

Rule 4 Choose matrixes which have the same structure as $\mathrm{T}_{\text {Aim }}$ or $\mathrm{C}_{\text {Aim }}$ to assemble if priority cannot be recognized so that unnecessary blank can be avoided as far as possible. This rule is also made to reduce the usage of cable and shorten cable length.

Step 7 After all matrix signals were allocated, do a final check to see if any adjustment can be done to get a better result.

\subsubsection{Improved greedy algorithm based matrix cable connection optimal design}

Definition $9 \mathrm{~S}_{\mathrm{R} \times \mathrm{C}}$ is the capacity of matrix cable allocation. 
Definition $10 \Delta$ Cost is the cost increment when choosing position.

Definition $11 \Delta$ Length is the length increment.

Definition $12 \mathrm{P}$ is insertion point. $\mathrm{P}$ is on the first line and first row of $\mathrm{T}_{\text {Aim }}$ or $\mathrm{C}_{\text {Aim }}$.

If $\mathrm{S}_{\mathrm{R} \times \mathrm{C}}=\left[\begin{array}{ccc}C & \cdots & C \\ \vdots & \ddots & \vdots \\ C & \cdots & C\end{array}\right]_{16 \times 24,16 \text { row lines and } 24}$ column lines can be shown in a sketch map as follows. In figure 1, judgment flags as TRUE or FALSE are set at junction point, such as $\mathrm{M}, \mathrm{N}, \mathrm{P}$.

According to figure 1, $\mathrm{C}_{\mathrm{Max}}=\left[\begin{array}{llll}C & C & C & C \\ C & C & C & C \\ C & C & C & C \\ C & C & C & C\end{array}\right]_{4 \times 4}$, $\left\{\mathrm{C}_{\mathrm{Next}}\right\} \quad$ has $\left[\begin{array}{ll}C_{\mathrm{N1}} & C_{\mathrm{N1}}\end{array}\right]_{1 \times 2} \quad$ and $\left[\begin{array}{ll}C_{N 2} & C_{N 2}\end{array}\right]_{1 \times 2}$. We can get $\left[\begin{array}{llll}C & C & C & C \\ C & C & C & C \\ C & C & C & C \\ C & C & C & C \\ C & C & C & C\end{array}\right]_{\text {to make }}$ $\Delta$ Cost least. Now, $\mathrm{C}_{\mathrm{Max}}=\left[\begin{array}{lll}C & C & C \\ C & C & C \\ C & C & C \\ C & C & C\end{array}\right]$. P has 10 probable $\left[\begin{array}{lllll}C & C & C & C & P \\ C & C & C & C & P \\ C & C & C & C & P \\ C & C & C & C & P \\ C & C & C & C & P \\ P & P & P & P & P\end{array}\right]$

positions as $\left.\begin{array}{lllll}P & P & P & P & P\end{array}\right]$. To make both $\Delta$ Cost and $\Delta$ Length least, the final position is $\left[\begin{array}{lllllll}C_{\text {Max }} & C_{\text {Max }} & C_{\text {Max }} & C_{\text {Max }} & C_{\text {Aim }} & C_{\text {Aim }} & C_{\text {Aim }} \\ C_{\text {Max }} & C_{\text {Max }} & C_{\text {Max }} & C_{\text {Max }} & C_{\text {Aim }} & C_{\text {Aim }} & C_{\text {Aim }} \\ C_{\text {Max }} & C_{\text {Max }} & C_{\text {Max }} & C_{\text {Max }} & C_{\text {Aim }} & C_{\text {Aim }} & C_{\text {Aim }} \\ C_{\text {Max }} & C_{\text {Max }} & C_{\text {Max }} & C_{\text {Max }} & C_{\text {Aim }} & C_{\text {Aim }} & C_{\text {Aim }} \\ C_{N 1} & C_{N 1} & C_{N 2} & C_{N 2} & & & \end{array}\right]$

Total cable length that $\mathrm{C}_{\text {Max }}$ used: $8 \times \mathrm{QA}=8 \times 8=64$

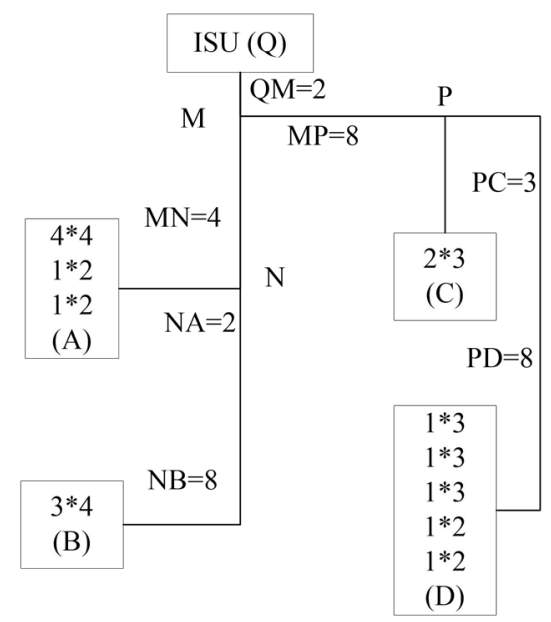

\section{Figure 1 Cable layout sketch map}

$\mathrm{C}_{\mathrm{N} 1}$ and $\mathrm{C}_{\mathrm{Max}}$ belong to the same device, they share same column lines. $\mathrm{C}_{\mathrm{N} 1}$ brings a new row line.So $\Delta$ Cost $_{=1}, \Delta$ Length $_{=} \mathrm{QA}=8$. Then $\mathrm{C}_{\mathrm{N} 2}$ and $\mathrm{C}_{\mathrm{Max}}$ belong to the same device, $\mathrm{C}_{\mathrm{N} 2}$ share the same column lines with $\mathrm{C}_{\mathrm{Max}}$, meanwhile, share the same row line with $\mathrm{C}_{\mathrm{N} 1}$. So $\Delta$ Cost $=0, \Delta$ Length $=0$ 。 Total length of $\mathrm{D}_{\text {Aim }}$ is $64+8=72$.

$$
\begin{aligned}
& \mathrm{C}_{\mathrm{Max}}=\left[\begin{array}{llll}
c & c & c & c \\
c & c & c & c \\
c & c & c & c \\
c & c & c & c
\end{array}\right] \quad . \quad \Delta \text { Cost } \quad=0+3=3 \quad, \\
& \Delta \text { Length }=4 \times \mathrm{NB}+3 \times \mathrm{BQ}=4 \times 8+3 \times 14=32+52=84 。
\end{aligned}
$$$$
\text { In }\left\{\mathrm{D}_{\mathrm{Next}}\right\} \text {, }
$$

After $\mathrm{D}_{\text {Aim }}$ is allocated, the judgment flags of $\mathrm{M}$ and $\mathrm{N}$ turned to TRUE as shown in figure 2 because these junction points have been used once. When moving to next step, algorithm searches the first TRUE point to the direction back to the driver. At last, we got $\begin{array}{ccccccc}C & C & C & C & C & C & C \\ C & C & C & C & C & C & C \\ C & C & C & C & C & C & C \\ C & C & C & C & C & C & C \\ C & C & C & C & C_{\operatorname{Max}} & C_{\operatorname{Max}} & C_{\operatorname{Max}} \\ & & & & C_{\text {Max }} & C_{\text {Max }} & C_{\text {Max. }} .\end{array}$

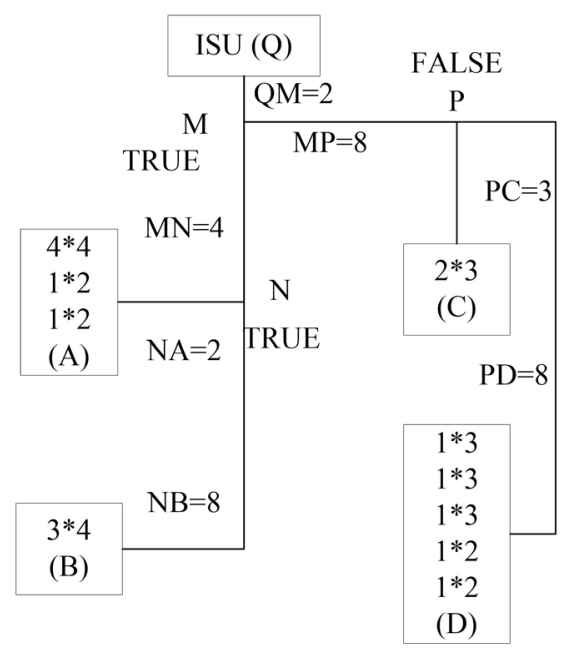

Figure 2 branch judgment flag

\section{Design example}

\subsection{Programming}

Interface 1 CountWidth

Interface 2 HeightComparator

Interface 3 Cell

Interface 4 SortCellUtil

The programming sturcture for interfaces is showed in figure 3.

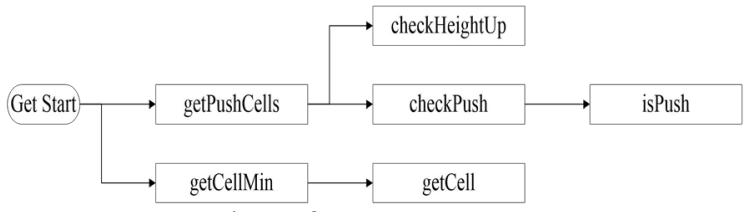

Figure 3 structure sketch 
The programming logic is showed in figure 4 .

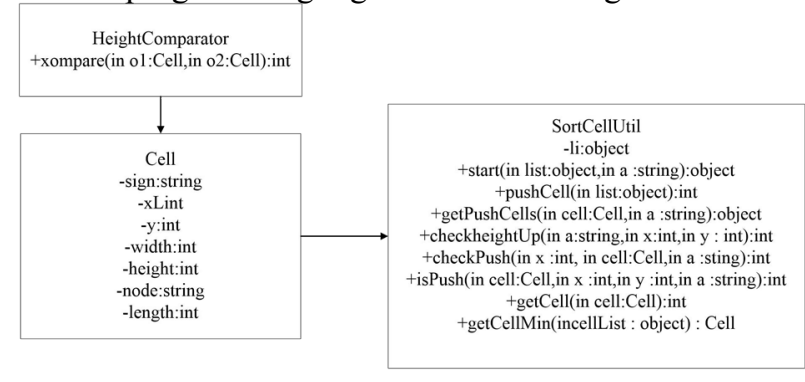

Figure 4 Programming logic

\subsection{Real project data verification}

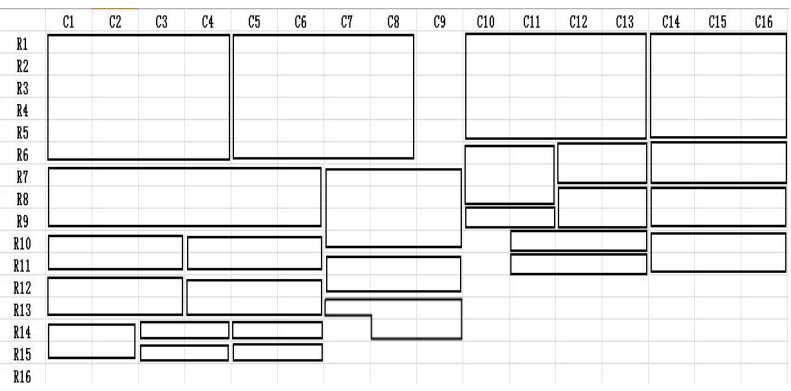

Figure 5 Optimal design result

Comparing with original design result, the optimal design result reduces total cable weight by $8 \%$ which is $0.416 \mathrm{~kg}$.

\section{Conclusion}

An improved greedy algorithm based matrix cable connection optimal design method is proposed in this article and the method is tested by real data project to prove its reasonableness and effectiveness. The result shows that optimal design result reduced weight by $8 \%$ comparing with original design result. Some complicated situation may occur in real projects such as branch terminals and layout adjustments. Later we will make further improvement of this optimal design method.

\section{Acknowledgments}

This work has been supported by Project of the Program of National Natural Science Foundation of China under the Grant Number 61471360.

This work has been supported by the 13th Five-Year Plan Pre-research special technology project from Space System Division.

\section{References}

1. Mingfeng Li, Yanyan Hao, Xiao Chen. New telecommunication satellite integrated electronic system research $[\mathrm{J}]$. Space International, 6, 46$50,(2013)$
2. Ke Zong, Zhifu Wang, Hangyan Xu. Optimal Mechatronic Design Research for Matrix Cables Used on Satellite [J]. Spacecraft Engineering,8(26),15-21 (2017)

3. Xiaoping Tang, Ruiqi Tian, and Martin D.F.Wong. Minimizing wire length in floorplanning[J]. IEEE Transaction on Computer-Aided Design of Integrated Circuits and Systems, 25(9), 17441753,(2006)

4. Gerhard Wäscher, Heike Haubner, Holger Schumann. An improved typology of cutting and packing problems [J]. European Journal of Operational Research, 183(3), 1109-1130,(2007)

5. Tolga Bektas, The multiple traveling salesman problem: An overviewof formulations and solution procedures [J]. Omega, 34(3),209-219,(2006)

6. Kris Braekers, Katrien Ramaekers, Inneke Van Nieuwenhuyse. The vehicle routing problem: State of the art classification and review [J]. Computers \& Industrial Engineering, 99,300-313,(2016) 